\title{
Análise do comportamento aplicada como intervenção para o autismo: definição, características e pressupostos filosóficos
}

\author{
Síglia Pimentel Höher Camargo* \\ Mandy Rispoli**
}

\begin{abstract}
Resumo
O transtorno do espectro do autismo (TEA) é um transtorno invasivo do desenvolvimento que persiste por toda a vida e não possui cura nem causas claramente conhecidas. No entanto, sabe-se que intervenções e métodos educacionais, com base na análise do comportamento aplicada (do inglês, Applied Behavior Analysis ou ABA), têm promovido uma variedade de habilidades sociais, acadêmicas, de comunicação e comportamentos adaptativos em indivíduos com TEA. O objetivo deste trabalho é apresentar a definição, características e pressupostos filosóficos que se encontram nas bases conceituais da ABA e fazem desta ciência um método de intervenção efetivo para pessoas com autismo.
\end{abstract}

Palavras-chave: ABA; Autismo; Intervenção.

* Professora Doutora da Universidade Federal de Pelotas (UFPel), Pelotas, Rio Grande do Sul, Brasil.

** Professor College of Education, Texas A\& University, College Station, TX, Estados Unidos da América do Norte (EUA). 


\title{
Applied behavior analysis as intervention for autism: definition, features and philosophical concepts
}

\begin{abstract}
Autism spectrum disorder (ASD) is a lifelong pervasive developmental disorder with no known causes and cure. However, educational and behavioral interventions with a foundation in applied behavior analysis (ABA) have been shown to improve a variety of skill areas such as communication, social, academic, and adaptive behaviors of individuals with ASD. The goal of this work is to present the definition, features and philosophical concepts that underlie ABA and make this science an effective intervention method for people with autism.
\end{abstract}

Keywords: ABA; Autism; Intervention.

\section{Introdução}

O transtorno do espectro do autismo (TEA) é caracterizado por alterações qualitativas nas habilidades de interação social, dificuldades de comunicação e o engajamento em comportamentos repetitivos e estereotipados (AMERICAN PSYCHIATRIC ASSOCIATION, 2000). O TEA pode afetar crianças de qualquer raça ou cultura e a expressão dos sintomas pode variar de leve a severo através dessas três áreas fundamentais (BERTOGLIO; HENDREN, 2009). Desse modo, os comportamentos, habilidades, preferências, funcionamento e necessidades de aprendizagem são diferentes de criança para criança e mudam ao longo do desenvolvimento (BOYD et al., 2008; LORD et al., 2000). Devido a variação na severidade dos sintomas, o transtorno do espectro do autismo representa um termo amplo que inclui, predominantemente, características diferentes de crianças com autismo clássico, síndrome de Asperger e transtorno invasivo do desenvolvimento sem outra especificação (AMERICAN PSYCHIATRIC ASSOCIATION, 2000; LEACH et al., 2009).

A prevalência de crianças diagnosticadas com TEA vêm crescendo em todo o mundo. Estatísticas recentes estimam que 1 em cada 50 crianças em idade escolar (6-12 anos) são diagnosticadas com autismo nos Estados Unidos (BLUMBERG et al., 2013; CENTER OF DISEASE CONTROL AND PREVENTION, 2013). No Brasil, não existe uma estimativa epidemiológica oficial (BRASIL, 2013), mas o número de brasileiros afetados pelo TEA também vêm aumentando, em parte pelo maior acesso à informações sobre o transtorno e à ferramentas de identificação precoce. O transtorno do espectro do autismo é um transtorno invasivo do desenvolvimento que persiste por toda a vida e não possui cura nem causas claramente conhecidas. No entanto, sabe-se que intervenções e métodos educacionais com base na psicologia comportamental têm demostrado reduzir os sintomas do espectro do autismo e promover uma 
variedade de habilidades sociais, de comunicação e comportamentos adaptativos. Esse método de intervenção e ensino é conhecido como a análise do comportamento aplicada ou ABA, sigla em inglês para Applied Behavior Analysis. (HOWARD et al., 2005; LANDA, 2007; VIRUES-ORTEGA, 2010; VISMARA; ROGERS, 2010).

Características gerais de uma intervenção baseada na ABA tipicamente envolvem identificação de comportamentos e habilidades que precisam ser melhorados (por exemplo, comunicação com pais e professores, interação social com pares, etc.), seguido por métodos sistemáticos de selecionar e escrever objetivos para, explicitamente, delinear uma intervenção envolvendo estratégias comportamentais exaustivamente estudadas e comprovadamente efetivas. Além disso, ABA é caracterizada pela coleta de dados antes, durante e depois da intervenção para analisar o progresso individual da criança e auxiliar na tomada de decisões em relação ao programa de intervenção e às estratégias que melhor promovem a aquisição de habilidades especificamente necessárias para cada criança (BAER, WOLF; RISLEY, 1968, 1987; HUNDERT, 2009). Por apresentar uma abordagem individualizada e altamente estruturada, ABA torna-se uma intervenção bem sucedida para crianças com TEA que tipicamente respondem bem à rotinas e diretrizes claras e planejadas (SCHOEN, 2003).

Sabe-se que o método ABA possui grande suporte científico e tem sido o método de intervenção mais pesquisado e amplamente adotado, sobretudo nos Estados Unidos, para promover a qualidade de vida de pessoas com transtorno do espectro do autismo (GILLIS \& BUTLER, 2007; LOVAAS, 1987; VAUGHN et al., 2003; VIRUÉS-ORTEGA, 2010; HOWARD et al., 2005; LANDA, 2007). No entanto, uma melhor e mais completa compreensão do ABA, enquanto método de intervenção em todas as suas dimensões e complexidade, requer o claro entendimento de sua base conceitual e dos princípios do comportamento que determinam a sua prática e fazem desta uma abordagem de intervenção efetiva, principalmente para pessoas com autismo. Portanto, nos propomos a seguir a introduzir brevemente, mas com maior profundidade, a definição, características e conceitos filosóficos que subjazem esta disciplina.

Inicialmente, a análise do comportamento aplicada pode ser definida como uma sistema teórico para a explicação e modificação do comportamento humano baseado em evidência empírica (HEFLIN; ALAIMO, 2007). Entretanto, uma completa definição da $\mathrm{ABA}$ requer o entendimento deste campo do conhecimento como uma abordagem científica, tecnológica e profissional. Como uma abordagem científica, ABA é definida como um método para avaliar, explicar e modicar comportamentos baseado nos princípios do condicionamento operante introduzidos por B.F. Skinner (SKINNER, 1953). Na perspectiva do condicionamento operante, os comportamentos são aprendidos no processo de interação entre o indivíduo e seu ambiente físico e social (SKINNER, 1953). Em outras palavras, o comportamento é influenciado pelos estímulos ambientais que o antecedem (chamados de antecedentes), e são aprendidos em função de suas consequências. Comportamentos que são seguidos por consequências que são especificamente agradáveis para o sujeito (por exemplo, atenção ou recompensa) tendem a ser repetidos e aprendidos, enquanto 
comportamentos que tem como consequência situações desagradáveis para o sujeito (por exemplo, uma reprimenda), tendem a não ser repetidos ou não aprendidos (ALBERTO; TROUTMAN, 2009). Considerando que esses princípios governam os comportamentos dos seres humanos, estes são entendidos como passíveis de predição, sendo que suas causas e funções podem ser identificadas nos eventos do ambiente (SKINNER, 1978). Portanto, ABA investiga as variáveis que afetam o comportamento humano, sendo capaz de mudá-los através da modificação de seus antecedentes (o que ocorreu antes e pode ter sido um possível gatilho para a ocorrência do comportamento) e suas consequências - eventos que se sucederam após a ocorrência do comportamento, e que podem ter sido agradáveis ou desagradáveis determinando a probabilidade de que ocorram novamente (SUGAI, LEWIS-PALMER; HAGANBURKE, 2000). Para estes propósitos, ABA usa métodos experimentais e sistemáticos de observação e mensuração dos comportamentos, os quais são definidos como aquelas ações dos indivíduos que são passíveis de serem observadas e mensuradas (MAYER et al., 2012). Ao medir comportamentos observáveis, ABA assume uma abordagem conduzida pelos dados na avaliação e intervenção de comportamentos que são importantes para os indivíduos e para a sociedade (BAER, WOLF, RISLEY, 1968). Portanto, enquanto uma abordagem científica, ABA utiliza princípios derivados de investigações científicas e demostra experimentalmente, através de dados empíricos consistentes, a eficácia dos procedimentos utilizados nas intervenções.

$\mathrm{Na}$ medida em que o conhecimento sobre como os comportamentos humanos são aprendidos e modificados são gradualmente produzidos em investigações experimentais, analistas do comportamento desenvolvem novos procedimentos e estratégias de intervenção para comportamentos que requerem atenção, tais como aqueles relacionados à habilidades acadêmicas, sociais e habilidades adaptativas de vida diária. Ao fornecer uma descrição específica, completa e cuidadosa de procedimentos baseados na evidência para modificar tais comportamentos, ABA é definida como uma tecnologia que é aplicada em situações de vida reais onde comportamentos apropriados e inapropriados podem ser melhorados, aumentados ou diminuídos.

Embora amplamente conhecida como um método de intervenção para pessoas com autismo (HOWARD et al., 2005; LANDA, 2007), ABA é uma tecnologia que pode ser aplicada à crianças e adultos com ou sem necessidades especiais em clínicas, escolas, hospitais, em casa, no ambiente de trabalho ou na comunidade (CAUTILLI, DZIEWOLSKA, 2008). Procedimentos usados pela ABA são baseados na avaliação detalhada das consequências que mantém os comportamentos de cada indivíduo e podem ser modificados, na medida em que a evidência demostra melhoras ou não ao longo do tempo e da intervenção. Cabe ressaltar com grande ênfase que os métodos e estratégias utilizadas na ABA não são baseadas em práticas aversivas para reduzir comportamentos indesejáveis. Embora estes procedimentos tenham sido estudados em experimentos com animais, a pesquisa atual tem enfatizado e demonstrado empiricamente que métodos baseados em técnicas de reforçamento positivo, que são consequências que motivam e aumentam a probabilidade de comportamentos desejáveis e adequados ocorrerem novamente, são mais efetivas e produzem melhoras mais significativas e duradouras do que métodos de punição, 
devendo portanto serem utilizadas em detrimento destes últimos (CAMERON, PIERCE, 1994; MAAG, 2001).

Considerando que a aplicação dos métodos da ABA requer treinamento apropriado, ABA pode ser também definida como uma abordagem profissional (MAYER et al., 2012). Analistas do comportamento são profissionais treinados para conduzir a análise do comportamento em sua dimensão, tanto experimental (através da pesquisa), quanto aplicada (através da intervenção). Os analistas do comportamento são orientados a utilizar intervenções efetivas, baseadas na evidência através de pesquisas experimentais controladas em casos envolvendo tanto comportamentos simples quanto complexos e possuem um código de princípios éticos fundamentais para guiar sua prática (BAILEY, BURCH, 2011). Nos Estados Unidos, o Behavior Analysis Certification Board é uma organização que representa e regulamenta a profissão, fornecendo certificação para profissionais que provem estar habilitados para desenvolver e aplicar intervenções baseadas nos princípios da análise dos comportamento. Embora nem todos os profissionais envolvidos com ensino e pesquisa em ABA, obrigatoriamente, tenham certificação do Board, a mesma tem sido exigida àqueles que oferecem serviços de intervenção para o público (CAUTILLI, DZIEWOLSKA, 2008; MAYER et al., 2012). Em outros países, como o Brasil, psicólogos são licenciados para trabalhar com a análise do comportamento, mas cabe ressaltar que estes devem buscar treinamento adicional e continuado de qualidade para atuar nessa área (TODOROV, HANNA, 2010).

As características científicas, tecnológicas e profissionais que definem a ABA acima descritas estão intimamente relacionadas com quatro pressupostos filosóficos, nos quais esta área do conhecimento se baseia: determinismo, empiricismo, parcimônia e método científico (ALBERTO, TROUTMAN, 2009; KIMBALL, 2002; MAYER et al., 2012). Estes pressupostos têm suas raízes nos movimentosfilosóficos do século XIX (a saber, positivismo, funcionalismo, estruturalismo e associacionismo), que enfatizavam que o comportamento humano deveria ser objetivamente estudado ao invés de abstratamente especulado (ALBERTO, TROUTMAN, 2009; KIMBALL, 2002). Tais pressupostos tiveram forte influência no behaviorismo radical - a filosofia do comportamento humano originado por B. F. Skinner em experimentos conduzidos sob o rigor dos métodos científicos (SMITH, 1992). Enquanto uma ciência derivada do trabalho de Skinner, ABA pode ser descrita pelos quatro pressupostos filosóficos que estão nas raízes do behaviorismo radical (LAMAL, 2000).

O determinismo é o pressuposto filosófico de que o comportamento humano é determinado ou causado pelos eventos do ambiente, portanto está sujeito à investigação científica e à predição, como qualquer outro fenômeno natural (LOCKE, 1964). Esta perspectiva da regularidade dos comportamentos é essencial na ABA que, baseada nos princípios do condicionamento operante, postula que a forma como seres humanos se comportam está diretamente e funcionalmente relacionada às consequências de suas ações (SUGAI, LEWIS-PALMER, HAGAN-BURKE, 2000). Devido ao fato de que essa perspectiva se mostra contrária às postulações filosóficas do livre arbítrio, em que seres humanos são considerados livres para decidir o curso de suas ações, técnicas comportamentais que alteram o comportamento humano são 
frequentemente criticadas como práticas coercivas e desumanas (AXELROD, 1996). Entretanto, o pressuposto de regularidade e leis que regem os comportamentos não indicam que ABA rejeita a liberdade humana (NEWMAN, REINECKE, KURTZ, 1996). Ao contrário, analistas do comportamento definem liberdade em termos da habilidade dos seres humanos de fazerem escolhas e do direito de exercitarem essa habilidade e terem opções (ALBERTO, TROUTMAN, 2009; BANDURA, 1975). O objetivo do analista do comportamento é aumentar as opções para o indivíduo com autismo, por exemplo, exercitar sua liberdade para escolher respostas alternativas a comportamentos mal-adaptativos (ALBERTO, TROUTMAN, 2009)

O empiricismo, enquanto outro conceito filosófico fundamental da ABA, postula que o conhecimento deve ser obtido a partir de fenômenos observáveis e mensuráveis, verificados pela experiência ou prática experimental (KIMBALL, 2002; KUBINA JR, FAN-YU, 2008). Enquanto uma ciência empírica, ABA conta com dados verificáveis obtidos através da observação sistemática de comportamentos como a fonte de conhecimento e técnicas produzidas.

Parcimônia refere-se ao pressuposto filosófico de que, quando duas teorias tentam explicar os mesmos fatos, aquela que é mais simples, mais breve, que faz suposições baseadas na observação, que pode ser mais facilmente explicada e tem maior probabilidade de generalidade, deve então ser considerada (EPSTEIN, 1984). Desse modo, parcimônia subjaz ABA, enquanto uma ciência empírica que explica comportamentos humanos, fornecendo conhecimento e estratégias sistemáticas, objetivas e concisas para a modificação do comportamento ao mesmo tempo que verifica a generalidade de suas suposições para diferentes pessoas, ambientes, culturas e comportamentos.

Por último, o método científico é um pressuposto filosófico que envolve um conjunto de técnicas controladas para empiricamente verificar hipóteses e estabelecer relações causais entre eventos (MAYER et al., 2012). Tanto a pesquisa básica, que tipicamente investiga princípios do comportamento em laboratórios, quanto a pesquisa aplicada, que investiga a aplicação desses princípios em ambientes e situações do cotidiano das pessoas, são baseadas no método científico. Embora experimentos altamente controlados sejam difíceis de serem conduzidos em situações cotidianas reais, ABA utiliza métodos de pesquisa de caso único (single case research) para delinear experimentos que possibilitam o controle de variáveis e obter conhecimento científico que são úteis para melhorar comportamento e a vida das pessoas.

Considerando os pressupostos filosóficos acima mencionados que se encontram nas bases conceituais da ABA, Baer, Wolf, Risley (1968) publicaram um artigo inaugural, apresentando sete dimensões da ABA na primeira edição do Journal of Applied Behavior Analysis (JABA). Estas dimensões são consideradas características fundamentais que definem e qualificam a análise do comportamento aplicada e que devem estar presentes em uma intervenção para que ela seja considerada ABA (HEFLIN, ALAIMO, 2007; MAYER et al., 2012). As dimensões são: aplicada, 
comportamental, analítica, tecnológica, conceitualmente sistemática, efetiva e generalidade. Cada uma destas dimensões será brevemente descrita abaixo.

Para ser considerada aplicada, uma intervenção deve focar comportamentos ou situações que são imediatamente importantes para o indivíduo e para a sociedade ao invés de importantes para teoria. Ao invés de estar interessada em comportamentos alimentares por que são importantes para o metabolismo, por exemplo, ABA está interessada neste comportamento devido a sua importância para a saúde e qualidade de vida das pessoas (BAER, WOLF, RISLEY, 1968). O objetivo final de uma intervenção considerada aplicada é tornar as pessoas mais independentes e socialmente ajustadas. Portanto, uma intervenção baseada na ABA deve ter validade social, isto é, deve vir ao encontro das necessidades dos indivíduos e da sociedade que devem estar satisfeitos com os procedimentos e resultados obtidos (WOLF, 1978).

Uma intervenção considerada comportamental é aquela preocupada com o que os indivíduos fazem ao invés do que eles dizem que fazem (BAER, WOLF, RISLEY, 1968). Isto significa que comportamentos devem ser observados e precisamente medidos, possibilitando avaliar a ocorrência de mudanças e a efetividade da intervenção. A precisão na mensuração de comportamentos pode ser um problema em estudos aplicados, porque torna-se necessário garantir que as mudanças realmente ocorreram no indivíduo observado e, não apenas, na percepção do observador. Para reduzir esse problema, analistas do comportamento utilizam medidas de confiabilidade para calcular o percentual de concordância entre dois ou mais observadores.

Em sua dimensão analítica, ABA requer a demonstração confiável dos eventos responsáveis pela ocorrência ou não-ocorrência dos comportamentos em estudo, permitindo assim a predição e controle das variáveis que afetam e mantém tais comportamentos (BAER, WOLF, RISLEY, 1968). Uma demonstração confiável envolve a replicação de medidas que consistentemente e repetidamente indicam certos procedimentos como responsáveis pelas mudanças observadas nos comportamentos. Demonstrações consistentes e controladas são geralmente obtidas através de designs de caso único (por exemplo, reversão experimental e linhas de base múltiplas) através das quais torna-se possível demonstrar e analisar relações causais entre os comportamentos e os eventos que os precederam ou sucederam.

A dimensão tecnológica da $\mathrm{ABA}$ refere-se à elaboração e definição operacional completa das estratégias e procedimentos que são efetivos para a aprendizagem e mudança de comportamentos (BAER, WOLF, RISLEY, 1968). Para ser considerada tecnológica, tanto a descrição do comportamento quanto dos procedimentos de intervenção devem ser claramente e objetivamente detalhados. A descrição de uma intervenção utilizando técnicas de reforçamento para comportamentos apropriados, por exemplo, precisam informar qual o tipo de reforço está sendo empregado, quem forncerá e quando será fornecido o reforço e o que será considerado comportamento apropriado (incluindo informações relevantes como frequência, intensidade e duração) para estabelecer a contingência entre o 
comportamento emitido e o reforço, como consequência deste comportamento. Descrições tecnológicas são características importantes da ABA por que permitem a aplicação e replicação dos procedimentos de intervenção utilizados.

Além de precisa, a descrição dos procedimentos da ABA deve ser conceitualmente sistemática (BAER, WOLF, RISLEY, 1968). Isso significa que os procedimentos devem estar relacionados com os princípios básicos do comportamento que as originaram. Procedimentos que fazem referência ao uso do reforço para aumentar a probabilidade de que comportamentos adequados ocorram, por exemplo, estão conceitualmente atrelados aos princípios do condicionamento operante. Este link entre a tecnologia e os conceitos básicos do comportamento são importantes, pois permitem que a análise aplicada do comportamento progrida como uma disciplina aplicada consistente.

Efetiva é outra característica essencial da ABA (BAER, WOLF, RISLEY, 1968). Os efeitos produzidos pelas técnicas comportamentais devem ser grandes o suficiente para produzir contribuições e mudanças importantes para a qualidade de vida do indivíduo e da sociedade. Novamente, isso refere-se a efeitos socialmente significativos pela sua importância prática ao invés de sua importância teórica. Portanto, uma análise do quão grande é uma mudança de comportamento é necessária para avaliar a efetividade de uma intervenção comportamental. Isto é possível através das consistentes coletas de dados ao longo da intervenção. No entanto, uma análise do tamanho da mudança ou do efeito da intervenção pode ser relativa e deve incluir pessoas que convivem diariamente com o comportamento alvo da intervenção, pois uma mudança aparentemente pequena de comportamento (por exemplo, aumento do repertório verbal de uma criança de 0 para 10 palavras) pode ser considerado significativo e socialmente importante.

A característica final da ABA descrita por Baer, Wolf, Risley (1968) é a generalidade. Intervenções comportamentais devem, não somente produzir mudanças socialmente importantes no comportamento, mas estas mudanças devem persistir através do tempo, dos ambientes e pessoas diferentes daquelas inicialmente envolvidas na intervenção. Uma intervenção que melhora a comunicação de uma criança com autismo na clínica, por exemplo, demonstra generalidade se a criança também consegue se comunicar com os pais, professores ou outras pessoas, em casa, na escola ou na comunidade, durante e após o término da intervenção. Entretanto, os autores enfatizam que a generalidade dos progressos comportamentais, não ocorrem automaticamente, sobretudo, em crianças com autismo que possuem dificuldades de transferir habilidades aprendidas para outros contextos. Portanto, a ocorrência de generalidade "deve ser programada e não esperada" (BAER, WOLF, RISLEY, 1968, p. 97).

A publicação das dimensões que caracterizam a $A B A$ constituiu e impulsionou o campo como uma ciência, tecnologia e profissão promissoras. As sete dimensões são importantes, não apenas por que descrevem, mas também por que guiam a análise do comportamento na produção de intervenções científicas que são baseadas na evidência e são úteis para a sociedade. Após a publicação de Baer, 
Wolf, Risley (1968) e a sua reanálise subsequente (BAER, WOLF, RISLEY, 1987), muitos estudos publicados são refinamentos de técnicas que venham ao encontro das características da ABA, acima mencionados, e melhorem a generalidade e manutenção dos resultados obtidos (HARVEY, LUISELLI, WONG, 2009), a validade social (WOLF, 1978), e a fidelidade de implementação da intervenção (PETERSON, HOMER, WONDERLICH, 1982), dentre outros aspectos. Portanto, estas dimensões são designadas a guiar a análise formativa da ABA, definindo critérios para a adequação da pesquisa e da prática, movendo o campo em direção à aplicação de intervenções mais efetivas (COOPER, HERON, HEWARD, 2007).

Desde a primeira publicação em 1968, ABA tem alcançado um crescimento notável, especialmente nos Estados Unidos, onde este campo de conhecimento foi originado. ABA está constantemente avançando para concretizar todas as dimensões que a tornam uma ciência respeitável. Muitas estratégias de pesquisa, avaliação e intervenção (por exemplo, designs de caso único, análise funcional do comportamento e estratégias de suporte comportamental positivos) foram desenvolvidas incorporando aspectos comportamentais, tecnológicos e conceituais que vem sendo utilizados como ferramentas valiosas para melhorar repertórios de comportamentos sociais, acadêmicos e de atividades de vida diária no cotidiano das pessoas (HORNER et al., 2005; IWATA, DORSEY, 1994; SUGAI, LEWIS-PALMER, HAGAN-BURKE, 2000).

No Brasil,ABA estágradualmenteganhandoespaçoenquantoum métodode intervenção para o autismo, mas somente poucos profissionais possuem treinamento apropriado na área. Os avanços da ABA enquanto uma ciência aplicada tem sido restritos no Brasil devido a uma maior ênfase em investigações e treinamento em pesquisa básica dos princípios do comportamento e pouco investimento em pesquisa e treinamento sobre a aplicação destes princípios para promover comportamentos socialmente importantes (TODOROV, HANNA, 2010). Ainda são necessários esforços da comunidade científica da análise do comportamento brasileiro para desenvolver a pesquisa e a prática no campo da análise do comportamento aplicada, em conformidade com as dimensões e princípios éticos que a constituem. Dessa forma, enquanto o campo gradativamente progride, tanto nos Estados Unidos quanto no Brasil, ABA cumprirá o seu papel de melhorar a qualidade de vida das pessoas, especialmente daquelas com transtornos do espectro do autismo.

\section{Referências}

ALBERTO, P. A.; TROUTMAN, A. C. Applied behavior analysis for teachers. 8th ed. Upper Saddle River, NJ: Pearson Education, Inc., 2009.

AMERICAN PSYCHIATRIC ASSOCIATION. Diagnostic and statistical manual of mental disorders (4th ed., text rev.). Washington, DC: Author, 2000.

AXELROD, S. What's wrong with behavior analysis? Journal of Behavioral Education. v. 6, n. 3, p. 247-247, 1996.

BAER, D. M.; WOLF, M. M.; RISLEY, T. R. Some current dimensions of applied behavior analysis. Journal of Applied Behavior Analysis, v. 1, n. 1, p. 91-97, 1968. 
BAER, D. M.; WOLF, M. M.; RISLEY, T. R. Some still-current dimensions of applied behavior analysis. Journal of Applied Behavior Analysis, v. 20, n. 4, p. 313-27, 1987.

BAILEY, J.; BURCH, M. Ethics for behavior analysts. 2.nd Expanded. New York: Routledge, 2011.

BANDURA, A. Annual review of behavior therapy, theory and practice. In: FRANKS, C. M.; WILSON, G. T. (Ed.), The ethics and social purposes of behavior modification, New York: Brunner/Mazel, 1975. p. 13-20.

BERTOGLIO, K.; HENDREN, R. New developments in autism. Psychiatric Clinics of North America, v. 32, n. 1, p. 1-1. 2009.

BLUMBERG, S.J.; BRAMLETT, M.D.; KOGAN, M.D.; ET AL. Changes in prevalence of parent-reported autism spectrum disorder in school-aged U.S. children: 2007 to 2011-2012. National health statistics reports. Hyattsville, MD: National Center for Health Statistics, 2013.

BOYD, B.; et al. Descriptive Analysis of Classroom Setting Events on the Social Behaviors of Children with Autism Spectrum Disorder. Education and Training in Developmental Disabilities, v. 43, n. 2, p. 186-197, 2008.

BRASIL. Secretaria de Atenção à Saúde. Departamento de Ações Programáticas Estratégicas. Diretrizes de Atenção à Reabilitação da Pessoa com Transtornos do Espectro do Autismo. Brasília: Ministério da Saúde, 2013.

CAMERON, J.; PIERCE, W. D. Reinforcement, reward, and intrinsic motivation: A metaanalysis. Review of Educational Research, v. 64, n. 3, p. 363-363, 1994.

CAUTILLI, J. D.; DZIEWOLSKA, H. Licensing behavior analysis. International Journal of Behavioral Consultation and Therapy, v. 4, n. 1, p. 1-13, 2008.

CENTERS FOR DISEASE CONTROL AND PREVENTION. Changes in prevalence of parent-reported Autism Spectrum Disorders (2013). Disponível em: 〈http://www.cdc.gov/ media/releases/2013/a0320_autism_disorder.html’. Acesso em: 24 abril 2013.

COOPER, J. O.; HERON, T. E.; HEWARD, W. L. Applied behavior analysis. 2.nd. Upper Saddle River, NJ: Pearson, 2007.

EPSTEIN, R. The principle of parsimony and some applications in psychology. Journal of Mind and Behavior, v. 5, n. 2, p. 119-130, 1984.

GILLIS, J. M.; BUTLER, R. C. Social skills interventions for preschoolers with Autism Spectrum Disorder: A description of single-subject design studies. Journal of Early \& Intensive Behavior Intervention, v. 4, n. 3, p. 532-547, 2007.

HARVEY, M. T.; LUISELLI, J. K.; WONG, S. E. Application of applied behavior analysis to mental health issues. Psychological Services, v. 6, n. 3, p. 212-222, 2009.

HEFLIN, L. J.; ALAIMO, D. F. Students with autism spectrum disorders: effective instructional practices. Upper Saddle River, NJ: Pearson Education, Inc., 2007.

HORNER, R. H.; et al. The use of single-subject research to identify evidence-based practice in special education. Exceptional Children, v. 71, n. 2, p. 165-179, 2005. 
HOWARD, J. S., et al. A comparison of intensive behavior analytic and eclectic treatments for young children with autism. Research in Developmental Disabilities, v. 26, n. 4, p. 359-383, 2005.

HOWARD, J. S.; et al. A comparison of intensive behavior analytic and eclectic treatments for young children with autism. Research in Developmental Disabilities, v. 26, n. 4, p. 359-383, 2005.

HUNDERT, J. Inclusion of students with autism: using ABA-based supports in general education. Austin: ProEd., 2009.

IWATA, B. A.; DORSEY, M. F. Toward a functional analysis of self-injury. Journal of Applied Behavior Analysis, v. 27, n. 2, p. 197-209, 1994.

KIMBALL, J. W. Behavior-analytic instruction for children with autism: philosophy matters. Focus on Autism \& Other Developmental Disabilities, v. 17, n. 2, p. 66-75, 2002.

KUBINA J. R.; RICHARD, M.; FAN-YU, L. Defining frequency: A natural scientific term. Behavior Analyst Today, v. 9, n. 2, p. 125-129, 2008.

LAMAL, P. A. The philosophical terrain of behavior analysis: a review of B. A. Thyer (ed.), The philosophical legacy of behaviorism. Journal of the Experimental Analysis of Behavior, v. 74, n. 2, p. 255-264, 2000.

LANDA, R. Early communication development and intervention for children with autism. Mental Retardation \& Developmental Disabilities Research Reviews, v. 13, n. 1, p. 16-25, 2007.

LEACH, D.; WITZEL, B.; FLOOD, B. Meeting the social communication needs of students with autism spectrum disorders across home and school settings. Focus on inclusive education, v. 6, n. 3, p. 1-7, 2009.

LOCKE, E. A. Determinism. American Psychologist, v. 19, n. 1l, p. 846-847, 1964.

LORD, C.; ET AL. Autism spectrum disorders. Neuron, v. 28, n. 2, p. 355-363, 2000.

LOVAAS, O. I. Behavioral Treatment and Normal Educational and Intellectual Functioning in Young Autistic Children. Journal of Consulting and Clinical Psychology, v. 55, n. 1, p. 3-9, 1987.

MAAG, J. W. Rewarded by punishment: reflections on the disuse of positive reinforcement in education. Exceptional Children, v. 67, n. 2, p. 173-86, 2001.

MAYER, G. R.; SULZER-AZAROFF, B.; WALLACE, M. D. Behavior analysis for lasting change. 2.nd. Cornwall-on-Hudson, NY: Sloan Publishing, LLC, 2012.

NEWMAN, B.; REINECKE, D. R.; KURTZ, A. L. Why be moral: humanist and behavioral perspectives. The Behavior Analyst, v. 19, n. 2, p. 273-280, 1996.

PETERSON, L.; HOMER, A. L.; WONDERLICH, S. A. The integrity of independent variables in behavior analysis. Journal of Applied Behavior Analysis, v. 15, n. 4, p. 477-92, 1982. 
SCHOEN, A. A. What Potential Does the Applied Behavior Analysis Approach Have for the Treatment of Children and Youth with Autism? Journal of Instructional Psychology, v. 30, n. 2, p. 125, 2003 .

SKINNER, B. F. Reflections on behaviorism and society. Englewood Cliffs: Prentice-Hall, 1978.

SKINNER, B. F. Science and human behavior. New York: Free Press, 1953.

SMITH, L. D. On prediction and control: B. F. Skinner and the technological ideal of science. The American Psychologist, v. 47, n. 2, p. 216-216, 1992.

SUGAI, G.; LEWIS-PALMER, T.; HAGAN-BURKE, S. Overview of the functional behavioral assessment process. Exceptionality, v. 8, n. 3, p. 149-60, 2000.

TODOROV, J. C.; HANNA, E. S. Análise do comportamento no Brasil. Psicologia: teoria e pesquisa, v. 26, p. 143-153, 2010.

VAUGHN, S.; et al. Social Skills Interventions for Young Children with Disabilities. Remedial $\&$ Special Education, v. 24, n. 1, p. 2, 2003.

VIRUES ORTEGA, J. Applied behavior analytic intervention for autism in early childhood: meta-analysis, meta-regression and dose-response meta-analysis of multiple outcomes. Clinical Psychology Review, v. 30, n. 4, p. 387-399, 2010.

VISMARA, L; ROGERS, S. Behavioral treatments in autism spectrum disorder: What do we know? Annual Review of Clinical Psychology, v. 6, p. 447-447, 2010.

WOLF, M. M. Social validity: the case for subjective measurement or how applied behavior analysis is finding its heart. Journal of Applied Behavior Analysis, v. 11, n. 2, p. 203-214, 1978.

\section{Correspondência}

Síglia Pimentel Höher Camargo - Universidade Federal de Pelotas, Unidades e Cursos de Graduação, Faculdade de Educação (FaE). Rua Coronel Alberto Rosa, Porto, CEP: 96010-770 - Pelotas, Rio Grande do Sul - Brasil.

E-mail: sigliahoher@yahoo.com.br

Recebido em 07 de fevereiro de 2013

Aprovado em 28 de maio de 2013 